\title{
MISTURAS DE FARINHA DE AVEIA E AMIDO DE MILHO COM ALTO TEOR DE AMILOPECTINA PARA PRODUÇÃO DE "SNACKS"1
}

\author{
Laura Beatriz KARAM², Maria Victória E. GROSSMANNN ${ }^{2, *}$, Rui Sérgio S. F. SILVA²
}

\begin{abstract}
RESUMO
O potencial de uso do amido modificado com alto teor de amilopectina combinado à farinha de aveia para produção de "snacks" extrusados foi investigado. O processamento foi executado em extrusor monorosca, de acordo com um delienamento fatorial $\left(2^{5}\right)$ incompleto, com 3 repetições no ponto central. As variáveis independentes controladas foram: umidade da matéria-prima, temperatura de extrusão, rotação do parafuso, diâmetro da matriz e teor de amido modificado. Como respostas, avaliou-se o volume específico, a dureza e a fraturabilidade. A partir da modelagem exploratória foram definidas as variáveis significativas e novos deslocamentos na área experimental, até se atingir o ponto ótimo para produção de "snacks". A melhor combinação de variáveis encontrada, para obter um produto com boas propriedades de expansão e de textura, foi: $17 \%$ de umidade de matéria-prima, $183^{\circ} \mathrm{C}$ de temperatura de extrusão, 100rpm de rotação do parafuso, $4 \mathrm{~mm}$ de diâmetro da matriz e $30 \%$ de amido modificado. O produto obtido nessas condições apresentou $7,2 \mathrm{~mL} / \mathrm{g}$ de volume específico, $5,41 \mathrm{~N}$ de dureza e $2,02 \mathrm{~N}$ de fraturabilidade, assemelhando-se, por essas características, aos produtos comerciais de milho.
\end{abstract}

Palavras-chave: "snacks"; aveia; amido modificado.

\section{SUMMARY}

OAT FLOUR/WAXY CORN STARCH BLENDS FOR SNACKS PRODUCTION. The potential application of modified starch to enhance properties of oat flour extruded products was investigated. The process was performed using a single screw extruder, applying a $2^{5}$ factorial design with 3 repetitions on the central point. The independent variables were: feed moisture, extrusion temperature, screw speed, die diameter and modified starch level and depended studied variables were: specific volume, hardness and fracturability. Based on results of the experiments a new experimental design was proposed to optimize product characteristics. The best combination of variables to obtain snacks with good expansion and texture was: $17 \%$ feed moisture, $183^{\circ} \mathrm{C}$ temperature, $100 \mathrm{rpm}$ screw speed, $4 \mathrm{~mm}$ die diameter and $30 \%$ waxy starch. The product obtained presented $7.2 \mathrm{~mL} / \mathrm{g}$ specific volume, $5.41 \mathrm{~N}$ hardness and $2.02 \mathrm{~N}$ fracturability, therefore their characteristics were similar to those of commercial corn products.

Keywords: snacks; oat; modified starch.

\section{1 - INTRODUÇÃO}

A aveia destaca-se dos outros cereais por fornecer um aporte energético e nutricional equilibrado, por conter em sua composição química aminoácidos, ácidos graxos, vitaminas e sais minerais indispensáveis ao organismo

${ }^{1}$ Recebido para publicação em 16/11/99. Aceito para publicação em 16/05/01.

2 UNIVERSIDADE ESTADUAL DE LONDRINA - Departamento de Tecnologia de Alimentos e Medicamentos. Cx. Postal 6001, CEP 86051-970, Campus Universitáio, Londrina/Pr, e-mail: victoria@uel.br

${ }^{*}$ A quem a correspondência deve ser enviada. humano e, principalmente, pela composição de fibras alimentares, que são de alta qualidade, como a $\beta$-glucana, uma fibra solúvel que possui efeitos hipocolesterolêmicos comprovados [22].

Os produtos de aveia existentes no mercado são limitados em número e desconhecidos quanto às formas de preparo pela maioria da população.

As indústrias que processam aveia produzem, para o consumo humano, o farelo obtido pela moagem da aveia e posterior separação da farinha por peneiragem e aspiração [23]. O farelo de aveia, segundo a definição da AMERICAN ASSOCIATION OF CEREAL CHEMISTS - AACC [2], deve conter: não mais de $50 \%$ da matéria-prima original (taxa de extração), no mínimo $5,5 \%$ de $\beta$-glucana (base seca), no mínimo $16 \%$ de fibra alimentar total (base seca) e, desta, no mínimo $33 \%$ deve ser solúvel.

Com a obtenção do farelo de aveia, surge um produto secundário, que é a farinha de aveia, apresentando maior teor de amido e menores teores de proteínas, lipídios e fibra alimentar, em relação aos da farinha integral [18], o que justificaria sua utilização na extrusão. GORDON et al [11] sugeriram o emprego da farinha de aveia na extrusão, pois os baixos níveis de fibras alimentares oferecem vantagens nas propriedades funcionais dos extrusados, quando comparados com os da farinha integral, como é o caso do aumento de expansão.

Nos testes preliminares que realizamos, porém, foi impossível extrusar a farinha de aveia, porque esta ficava compactada impedindo a rotação do parafuso.

De acordo com EXTRUDED [10], os extrusados de aveia são densos, duros e de baixa expansão e uma maneira de melhorar essas características negativas seria combinar a farinha de aveia com amidos modificados. Não há porém informações quanto às condições operacionais e propriedades desses extrusados. Diante disso, o objetivo deste trabalho foi avaliar o comportamento da farinha de aveia combinada ao amido modificado com elevado teor de amilipectina para produção de "snacks" extrusados.

\section{2 - MATERIAL E MÉTODOS}

\section{1 - Material}

Os "snacks" foram produzidos a partir da farinha de aveia (Avena sativa L.), cultivar IAC 7 cuja composição centesimal apresentou: $13,4 \%$ de proteína, 7,9\% de lipídios, $4,0 \%$ de fibra alimentar e $63 \%$ de carboidratos, segundo os métodos da AACC [1], fornecida pela S. L. Cereais e Alimentos Ltda. (Mauá da Serra - Pr) e do 
amido modificado, BAKA SNAK um amido ceroso de milho, composto por $99 \%$ de amilopectina, prégelatinizado (informações do fabricante), fornecido pela National Starch \& Chemical Industrial Ltda (São Paulo $-\mathrm{SP})$.

\section{2 - Métodos analíticos}

\subsection{1 - Preparo e condicionamento da matéria- prima}

Para a produção de "snacks", o amido modificado foi incorporado à farinha de aveia, nas proporções determinadas pelo delineamento experimental, adicionandose água até atingir o teor de umidade estabelecido pelo mesmo delineamento. O material foi acondicionado em sacos de polietilieno de baixa densidade que, devidamente selados, foram armazenados em geladeira por uma noite, para atingir o equilíbrio.

\subsection{2 - Extrusão}

Foi executada em extrusor de laboratório CT-L15 (CEREALTEC INTERNATIONAL, Campinas, Brasil), tipo monorosca, com parafuso de $420 \mathrm{~mm}$ de comprimento, $19,4 \mathrm{~mm}$ de diâmetro e taxa de compressão 1:2. A alimentação do material no extrusor atingiu $0,09 \mathrm{~kg} \cdot \mathrm{min}^{-1}$. A temperatura da primeira zona de aquecimento foi fixada em $80^{\circ} \mathrm{C}$, enquanto que a rotação do parafuso, o diâmetro da matriz e a temperatura na segunda e terceira zona e na matriz variaram conforme o nível estabelecido no delineamento. Os "snacks" foram coletados após o processo atingir o seu equilílbrio, determinado pela estabilidade da amperagem no extrusor. A seguir, foram secos a $100^{\circ} \mathrm{C} / 15 \mathrm{~min}$, em estufa com circulação de ar, para reduzir o conteúdo de umidade até valores próximos a $3 \%$.

\subsection{3 - Propriedades dos "snacks"}

O volume específico $(\mathrm{mL} / \mathrm{g})$ foi determinado, em triplicata, pela técnica de deslocamento de areia [14]. A dureza $(\mathrm{N})$ e fraturabilidade $(\mathrm{N})$ foram determinadas utilizando o Texturômetro TA.XT2i (Texture Techologies Corp., Scarsdale, NY), auxiliado pelo software XTRAD. Vinte amostras de tamanho similar foram unixialmente cortadas com ponta de prova HDP/BSK (Blade Set with Knife), com velocidade de $5,0 \mathrm{~mm} / \mathrm{s} ; 20,0 \mathrm{~mm}$ de distância; limiar de força $0,20 \mathrm{~N}$ e limiar de distância $0,5 \mathrm{~mm}$.

\subsection{4 - Delineamento experimental}

\section{Modelagem exploratória}

Antecedendo a extrusão, foi definido um delineamento fatorial $\left(2^{5}\right)$, contendo 5 variáveis em 2 níveis eqüidistantes $(-1$ e +1$)$ acrescido de três repetições no ponto central (nível zero), através do software Statistica for Windows versão 4.3 [20], resultando em 11 ensaios com uma otimalidade $D=95,88 \%$. As cinco variáveis independentes foram: $U M=$ teor de umidade da matéria-prima $(17,20,23 \%)$, TEM = temperatura de extrusão $\left(120,150\right.$ e $\left.180^{\circ} \mathrm{C}\right)$, ROT = rotação do parafuso $(100$,
140 e 180rpm), MAT = diâmetro da matriz $(4,5$ e $6 \mathrm{~mm})$ e $\mathrm{AMI}=$ teor de amido modificado $(10,20$ e $30 \%)$. O teor zero de amido não foi incluído porque a farinha de aveia pura não fluiu no extrusor.

\section{Modelagem para otimização}

A partir dos resultados do ensaio exploratório foi realizado um deslocamento do delineamento experimental para região de baixa umidade (16-18\%) e alta temperatura $\left(165-195^{\circ} \mathrm{C}\right)$, empregando-se delineamento composto rotacional, com as 2 variáveis codificadas nos níveis: $-\alpha,-1,0,+1,+\alpha$, onde $\alpha=2^{1 / 2}$ [4]. A rotação do parafuso, diâmetro da matriz e teor de amido modificado foram mantidos constantes, nos níveis $-1,-1,+1$, respectivamente.

\subsection{5 - Análise estatística}

As propriedades analisadas em cada ensaio: VE= volume específico, DU= dureza e FRA= fraturabilidade foram avaliadas, inicialmente, através de análise de variância (ANOVA) para determinar: a significância da regressão ao nível de $5 \%$ (teste $\mathrm{F}$ ), o coeficiente de variação $(C V)$ e o coeficiente de determinação $\left(R^{2}\right)$, usando os softwares Statistical Analysis System [19] e Planej [4].

O procedimento "stepwise" [19], foi utilizado para eliminação das variáveis não significativas $(p<0,05)$ e simplificação dos modelos. Gráficos tridimensionais foram elaborados a partir das equações ajustadas, utilizando o software Statística for Windows 4.3 [20].

\section{3 - RESULTADOS E DISCUSSÃO}

A Tabela 1 mostra o delineamento experimental e os valores médios obtidos para o volume específico, dureza e fraturabilidade dos extrusados. Na Tabela 2, são apresentados os modelos de regressão ajustados a esses dados experimentais.

TABELA 1. Valores médios do volume específico, dureza e fraturabilidade dos extrusados, segundo o delineamento fatorial $2^{5}$ incompleto

\begin{tabular}{ccccccccc}
\hline Ensaios $^{*}$ & \multicolumn{4}{c}{ Variáveis Independentes(valores reais) } & \multicolumn{3}{c}{ Variáveis Resposta } \\
\hline & UM & TEM & ROT & MAT & AMl & VE & DU & FRA \\
\hline 1 & 23 & 180 & 180 & 6 & 30 & 3,0 & 8,942 & 4,895 \\
2 & 23 & 180 & 100 & 6 & 10 & 4,1 & 3,134 & 0,721 \\
3 & 23 & 120 & 180 & 4 & 30 & 1,8 & 24,342 & 9,258 \\
4 & 23 & 120 & 100 & 4 & 10 & 1,3 & 25,660 & 15,140 \\
5 & 17 & 180 & 180 & 4 & 10 & 1,3 & 10,354 & 8,438 \\
6 & 17 & 180 & 100 & 4 & 30 & 4,0 & 17,130 & 11,465 \\
7 & 17 & 120 & 180 & 6 & 10 & 1,0 & 47,777 & 6,073 \\
8 & 17 & 120 & 100 & 6 & 30 & 0,8 & 66,959 & 29,907 \\
9 & 20 & 150 & 140 & 5 & 20 & 1,1 & 24,944 & 19.707 \\
10 & 20 & 150 & 140 & 5 & 20 & 1,0 & 16,357 & 13,637 \\
11 & 20 & 150 & 140 & 5 & 20 & 1,0 & 29,812 & 28,178 \\
\hline
\end{tabular}

UM= Umidade $(\%), \operatorname{TEM}=$ Temperatura $\left({ }^{\circ} \mathrm{C}\right), \mathrm{ROT}=$ rotação $(\mathrm{rpm}), \mathrm{MAT}=\operatorname{Matriz}(\mathrm{mm})$, $\mathrm{AMI}=$ Amido modificado $(\%), \mathrm{VE}=$ Volume específico $(\mathrm{mL} / \mathrm{g}), \mathrm{DU}=$ Dureza $(\mathrm{N})$, $\mathrm{FRA}=$ Fraturabilidade $(\mathrm{N}),{ }^{*}$ realizados em ordem aleatória 
A análise de variância (ANOVA) mostrou que esses modelos são significativos $(p<0,05)$. Os coeficientes de determinação $\left(R^{2}\right)$ foram altos $(>0,90)$ para volume específico e dureza. No caso do primeiro a falta de ajuste não foi significativa e o coeficiente de variação foi baixo $(12,4 \%)$, podendo ser considerado como um instrumento preditivo, enquanto o segundo pode apenas indicar a tendência da resposta, por ter apresentado falta de ajuste significativa. Para a fraturabilidade, o $\mathrm{R}^{2}$ foi mais baixo, explicando $73,61 \%$ da variação da resposta e a falta de ajuste não foi significativa. O coeficiente de variação (CV) apresentou-se relativamente elevado $(39,73 \%)$, mas isto é aceitável para esta resposta, atribuindo-se boa parte dessa variação às características heterogêneas de textura dos produtos e à alta sensibilidade do equipamento. A equação ajustada foi utilizada para prever a resposta na região analisada.

TABELA 2. Modelos de regressão ajustados aos dados experimentais segundo o delineamento fatorial $2^{5}$ incompleto para volume específico, dureza e fraturabilidade dos extrusados.

\begin{tabular}{|c|c|c|}
\hline Propriedade & Modelo de Regressão & $\mathbf{R}^{2}$ \\
\hline VOLUME ESPECÍFICO & $\begin{array}{l}V E=1,032+0,405 \text { UM + 0,926 TEM - 0,391 } \\
R O T+0,237 \text { AMI }+1,134 \text { UM }^{2}-0,568 \text { TEM } \\
R O T\end{array}$ & 0,9863 \\
\hline DUREZA & $\begin{array}{l}D U=24,944-10,021 U M-15,650 \text { TEM + } \\
6,163 \text { MAT }\end{array}$ & 0,9066 \\
\hline FRATURABILIDADE & $F R A=16,129-6,984$ UM - 8,107 TEM & 0,7361 \\
\hline
\end{tabular}

$\mathrm{UM}=$ valores codificados para umidade TEM $=$ valores codificados para temperatura; $\mathrm{ROT}$ = valores codificados para rotação, $\mathrm{AMI}=$ valores codificados para amido modificado e MAT = valores codificados para matriz

\section{1 - Volume específico}

O volume específico foi afetado por todas as variáveis estudadas, com exceção da matriz. Esse é um parâmetro primário, associado às medidas de fraturabilidade, dureza, absorção de água e solubilidade em água dos produtos extrusados produzidos por extrusão termoplástica. Está diretamente relacionado com a expansão (axial + radial) e negativamente correlacionado com a densidade [3].

No gráfico de efeito da temperatura e umidade no volume específico, apresentado na Figura $1 \mathrm{~A}$, onde as variáveis ROT e AMI foram fixadas no nível zero (140rpm e $20 \%$, respectivamente), podemos observar que a tendência para maiores valores de volume específico se localiza na região de maior temperatura de extrusão $\left(180^{\circ} \mathrm{C}\right)$ e maior de umidade da matéria-prima $(23 \%)$. Vários pesquisadores vêm estudando o efeito do teor de umidade da matéria-prima e da temperatura de extrusão nas propriedades dos extrusados [12, 16, 17]. A umidade afeta significativamente a expansão e a força de quebra dos extrusados e foi considerada a variável mais importante do processo [8]. Um baixo teor de umidade do amido pode restringir o fluxo do material dentro do extrusor aumentando, assim, a taxa de cisaIhamento e tempo de residência, o que, provavelmente, aumenta o grau de gelatinização do amido e, por conseguinte, a expansão. Todavia, quando o teor de umidade é baixo demais (inferior a 15\%), isto pode criar uma taxa de cisalhamento muito alta, aumentando a temperatura do produto e conseqüentemente, a degradação do amido. Tal condição pode resultar na redução da expansão $[9,13]$. De acordo com VILELA \& ELDASH [21], o aumento da temperatura de extrusão diminui a viscosidade do gel, provocando um maior fluxo do material durante a extrusão, maior porosidade e, consequentemente, menor densidade do extrusado.
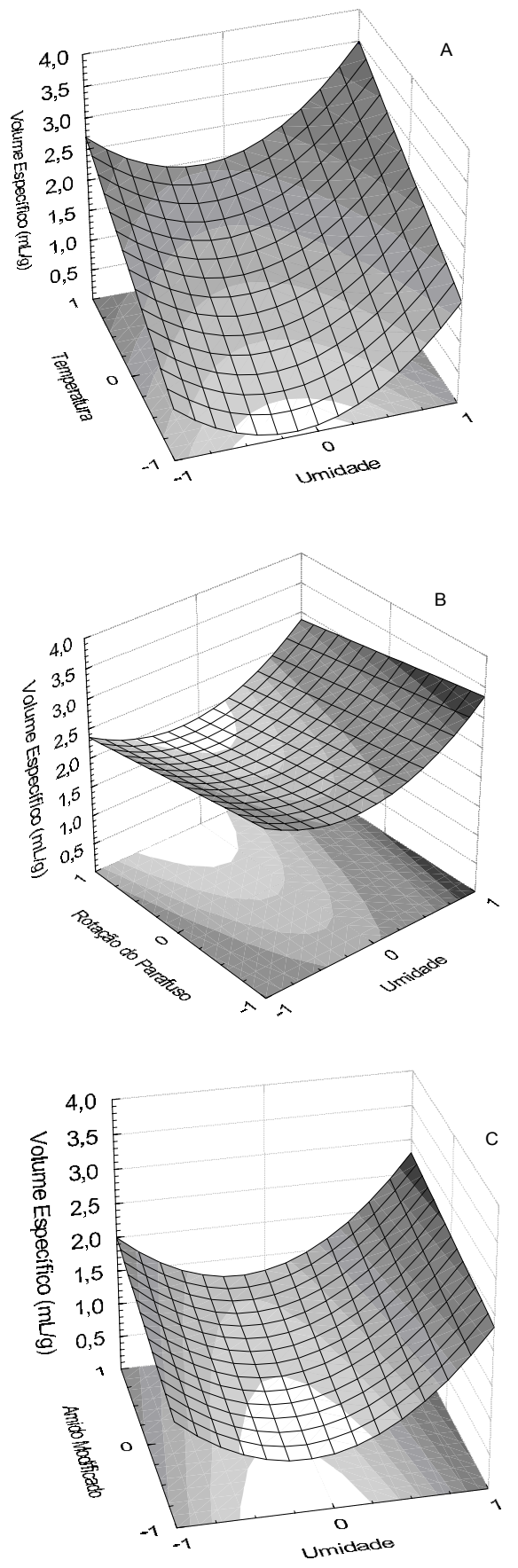

FIGURA 1. Efeito de: (A) temperatura e umidade, $(B)$ rotação do parafuso e umidade e $(C)$ teor de amido e umidade sobre o volume específico dos extrusados. (As variáveis que não aparecem nos gráficos foram mantidas fixas no nível zero). 
Analisando a Figura 1B, onde TEM e AMI foram fixados no nível zero $\left(150^{\circ} \mathrm{C}\right.$ e $20 \%$, respectivamente), observou-se que, quanto menor a rotação do parafuso (100rpm), para os mesmos teores de umidade, maiores valores para volume específico podem ser alcançados. Com a diminuição da rotação do parafuso, diminui-se o cisalhamento, mas aumenta-se o tempo de residência, o que pode ter contribuído para atingir um grau de gelatinização ideal para aumentar o volume [5]. Segundo HARPER [13], durante a extrusão, muita da energia necessária para alcançar a temperatura desejada vem da dissipação da energia mecânica para rotacionar o parafuso. $O$ controle da temperatura e a rotação do parafuso tem um profundo efeito nas condições de expansão do extrusado.

De acordo com a Figura $1 \mathrm{C}$, onde foram fixadas as variáveis TEM $\left(150^{\circ} \mathrm{C}\right)$ e ROT (140rpm), o amido modificado apresentou maior influência sobre o aumento do volume específico com a umidade no nível mais baixo (17\%), do que com a umidade no nível mais alto (23\%). $\mathrm{Na}$ aveia, a fração de amilose varia de 19 a 27\%, sendo o restante amilopectina [6]. Com a adição de $30 \%$ de BAKA SNAK ( $99 \%$ de amilopectina) a concentração de amilose reduziu consideravelmente, o que, segundo HARPER [13], contribui nas propriedades de expansão, resultando em um extrusado com maior volume específico. Além da amilose, outros componentes reduziram proporcionalmente, como os lipídios, que, agora com valores próximos a $5 \%$, teriam um efeito menor na redução da expansão [7].

Avaliando os efeitos das variáveis independentes TEM (Figura 1A), ROT (Figura 1B) e AMI (Figura 1C), relacionados ao teor de umidade, observa-se que as interações apresentaram maiores valores para volume específico quando a umidade seguia a tendência ao valor máximo (23\%), embora houvesse, também, uma tendência para aumento do volume específico em teores mínimos de umidade $(17 \%)$. Nas condições de alta umidade, porém, o fluxo de material no extrusor apresentava descontinuidade, prejudicando as características de aparência, formato e estrutura dos "snacks". Assim as condições de menor umidade se apresentaram como mais satisfatórias.

\section{2 - Dureza e fraturabilidade}

A dureza dos extrusados diminuiu em função de TEM e UM e aumentou em função de MAT (Tabela 2). As melhores condições para obtenção de extrusados com baixa dureza foram: $23 \%$ de umidade, $180^{\circ} \mathrm{C}$ de temperatura e $4 \mathrm{~mm}$ de diâmetro da matriz (Figura 2). Estes parâmetros estão em concordância com aqueles necessários para obter um volume específico elevado, considerado uma propriedade física decisiva na qualidade de "snacks".

A fraturabilidade foi influenciada significativamente apenas pela umidade e temperatura (Tabela 2). Valores mais baixos de fraturabilidade foram atingidos quando utilizados maiores níveis de temperatura e umidade, $180^{\circ} \mathrm{C}$ e $23 \%$, respectivamente (Figura 3).
O que se deseja em "snacks" com boas características de textura é que os valores de dureza e fraturabilidade sejam baixos. MENDONÇA, KARAM, GROSSMANN [15], estudando as propriedades de textura de "snacks" comerciais de milho, encontraram valores que variaram de 7,35 a $11,5 \mathrm{~N}$ e 4,70 a $5,70 \mathrm{~N}$ para dureza e fraturabilidade, respectivamente. Assim, mesmo que as condições de processamento utilizadas fossem as de menor umidade e maior temperatura (apontadas anteriomente como mais recomendadas para o volume específico) os valores de dureza e fraturabilidade ficariam dentro da faixa encontrada em "snacks" tradicionais.

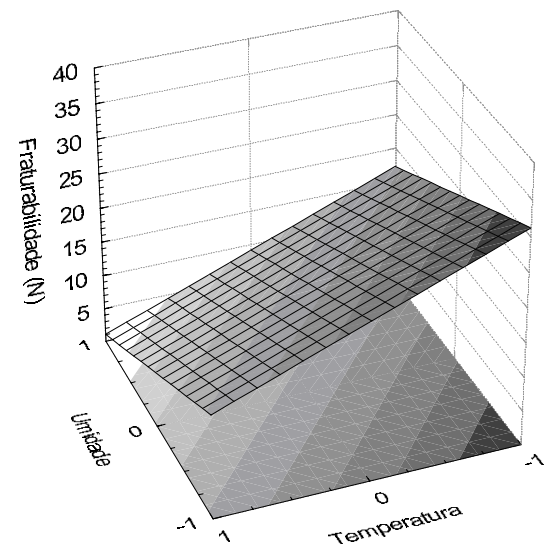

FIGURA 2. Efeito da umidade da matéria-prima e temperatura de extrusão na dureza dos "snacks" (matriz $=4 \mathrm{~mm}$ )

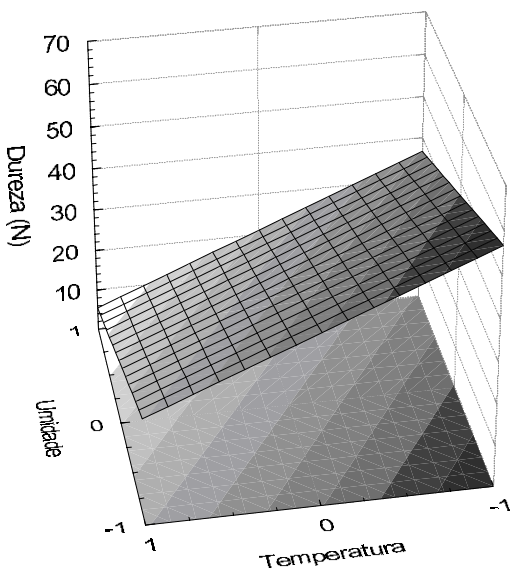

FIGURA 3. Efeito da umidade da matéria-prima e temperatura de extrusão sobre a fraturabilidade dos extrusados

\section{3 - Otimização da produção de "snacks"}

Com o intuito de otimizar as condições para produção de "snacks" foi definido um novo delineamento, levando em consideração o comportamento das variáveis independentes em relação ao volume específico, por ser esta variável a que mais se correlaciona com a expansão e textura desses produtos. O modelo de regressão obtido no delineamento $2^{5}$ incompleto, indicou 
que maiores valores de volume específico podem ser obtidos com a redução da rotação do parafuso e com o aumento da temperatura de extrusão, do teor de amido e da umidade; sendo que menores níveis de umidade também apresentaram aumento no volume específico. Pelos problemas já relatados em relação ao efeito da alta umidade (Item 3.1), optou-se por explorar a região de baixa umidade e alta temperatura. Devido às limitações do extrusor (mono-rosca), a rotação mínima que pôde ser executada para esse tipo de matéria-prima foi $100 \mathrm{rpm}$, por isso foi fixada essa condição. Considerando-se, também, que o objetivo do trabalho foi produzir "snacks" com maior teor possível de farinha de aveia, optou-se por estudar o efeito do amido modificado (BAKA SNAK) na concentração máxima de $30 \%$. O diâmetro da matriz não foi significativo no aumento de volume específico, mas analisando os parâmetros de dureza optou-se por fixá-lo em $4 \mathrm{~mm}$, porque os "snacks" produzidos com essa matriz apresentaram-se menos duros.

Realizados os ensaios do delineamento para otimização, o modelo de regressão ajustado aos dados experimentais para volume específico:

VE $=7,150+0,347$ UM + 0,530 TEM - 0,963 UM ${ }^{2}-1,063$ TEM $^{2}$

foi significativo $(\mathrm{p}<0,05)$ e explicou $88,21 \%$ da variação da resposta. Na Figura 4, observa-se que o maior valor dessa resposta $(7,2 \mathrm{~mL} / \mathrm{g})$, apontado pela análise estatística dos resultados, pode ser obtido com $17 \%$ de umidade de matéria-prima e $183^{\circ} \mathrm{C}$ de temperatura de extrusão. Ou seja, ao se deslocar a região experimental seguindo o percurso apontado pelo modelo inicial, encontrou-se uma região operacional ótima.

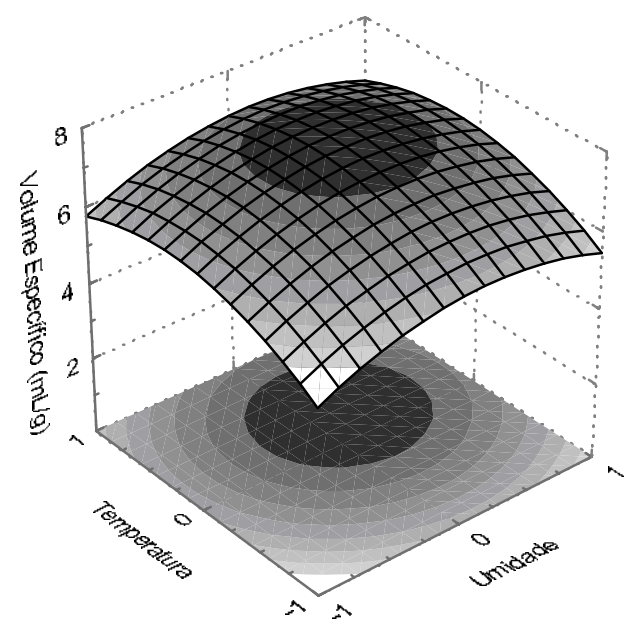

FIGURA 4. Volume específico dos "snacks", em função da umidade da matéria-prima e da temperatura de extrusão (experimento de otimização).

Os "snacks" obtidos nessas condições tiveram valores médios de $5,41 \mathrm{~N}$ e $2,02 \mathrm{~N}$, para dureza e fraturabilidade, respectivamente. Estes são inferiores aos verificados em "snacks" comerciais de milho [15], mas perfeitamente aceitáveis, conferindo aos produtos características excelentes de crocância e palatabilidade (não quantificadas).

\section{4 - CONCLUSÕES}

A adição de amido de milho com alto teor de amilopectina à farinha de aveia possibilitou a produção de "snacks" com boas propriedades de expansão, dureza e fraturabilidade. A metodologia de superfície de resposta permitiu a definição das variáveis relevantes e as condições ótimas de processamento, definidas em: $30 \%$ de amido de milho ceroso, $17 \%$ de umidade de matéria-prima, $183^{\circ} \mathrm{C}$ de temperatura de extrusão, $100 \mathrm{rpm}$ de rotação do parafuso e matriz de $4 \mathrm{~mm}$.

\section{5 - REFERÊNCIAS BIBLIOGRÁFICAS}

[1] AACC - AMERICAN ASSOCIATION OF CEREAL CHEMISTS. Approved methods, 8. ed. Saint Paul, 1983.

[2] AACC - AMERICAN ASSOCIATION OF CEREAL CHEMISTS. Comittee adopts oat bran definition. Cereal Food World, Saint Paul, v. 34, n. 12 p. 1034, 1989.

[3] ALI, Y., HANNA, M. A. \& CHINNASWAMY, R. Expansion characteristics of extruded corn grits. LebensmittelWissenschaft-und-Technologie, London, v. 29, n. 8, p. 702707, 1996.

[4] BARROS NETO, B., SACARMINIO, I. S. \& BRUNS R. E. Planejamento e otimização de experimentos. Campinas: UNICAMP, 299p., 1995.

[5] BATTACHARYA, M. \& HANNA, M. A. Textural properties of extrusion-cooked corn starch. LebensmittelWissenschaft-und-Technologie, London, v. 20, n. 4, p. 195-207, 1987.

[6] BECKER, R. \& HANNERS, G. Carbohydrate composition of cereal grains. In: LORENZ, K. J. \& KULP, K. Handbook of Cereal Science and Technology, New York: Marcel Dekker, 1991. p. 469-496.

[7] CHEFTEL, J. C. Nutritional effects of extrusion-cooking. Food Chemistry, Oxford, v. 20, n. 4, p. 263-283, 1986.

[8] CHEN, J., SERAFIN, F. L., PANDYA, R.N. \& DAUN, H. Effects of extrusion conditions on sensory properties of corn meal extrudates. Journal of Food Science, Chicago, v. 56, n. 1, p. 84-89, 1991

[9] CHINNASWANNY, R. \& HANNA, M. A. Optimum extrusioncooking conditons for maximum expansion of corn starch. Journal of Food Science, Chicago, v. 53, n. 3, p. 834836, 840, 1988.

[10] EXTRUDED snackfoods: starch to the rescue. Food Manufacture, London, v. 65, n. 9, p. 21-23, 1990.

[11] GORDON, W. A., HEMPENIUS, W. L. \& KIRKWOOD, J. R. Process for preparing a highly expanded oat cereal products. U.S. patent:: 4.620.981,1986.

[12] HARPER, J. M. Extrusion of Food. Boca Raton: CRC Press, V. 1, 1981. 212p.

[13] HARPER, J. M. Extrusion texturization of foods. Food Technology, Chicago, v. 40, n. 3, p. 70-76, 1986.

[14] LUE, S., HSIEH, F. \& HUFF H. E. Extrusion cooking of corn meal and sugar beet fiber: effects on expansion properties, starch gelatinization, and dietary fiber content Cereal Chemistry, Saint Paul, v. 68, n. 3, p. 227-234, 1991.

[15] MENDONÇA S.; KARAM, L. B.; GROSSMANN, M. V. E. Análise Instrumental de textura e sua correlação com a avalia- 
ção sensorial em "snacks" comerciais. XVI CONGRESSO BRASILEIRO DE CIÊNCIA E TECNOLOGIA DE ALIMENTOS. Rio de Janeiro, R. J. Anais. v. 1, p. 84-87, 1998.

[16] MERCIER, C. \& FEILLET, P. Modification of carbohydrate components by extrusion-cooking of cereal products. Cereal Chemistry, Saint Paul, v. 52, n. 3, p. 283-297, 1975.

[17] MILLER, R.C. Extrusion cooking of pet foods Cereal Foods World, Saint Paul, v. 30, p. 323-327, 1985.

[18] PATON, D. \& LENZ, M. K. Current practice and novel processes. In: WOOD, P. J. Oat Bran. Saint Paul: American Association of Cereal Chemists, p. 25-48. 1993.

[19] STATISTICAL ANALYSIS SYSTEM. SAS/QC $®$ Software: Reference. 5 ed. Cary, 1985. 956p.

[20] STATISTICAL FOR WINDOWS. GRAPHICS SOFTWARE: STATSOFT, Tulsa, Oklahoma, 1995.
[21] VILELA, E. R. \& EL-DASH, A. A. Extrusão de farinha de gandu (Cajanus cajan, Mill sp.). I. Efeitos das variáveis do processo nas características químicas, físicas e físico-químicas dos produtos extrusados. Ciênc. Tecnol. Aliment., Campinas, v. 7, n. 2, p. 97-116, 1987.

[22] WEBSTER, F. H. Oat utilization: past, present, and future. In: WEBSTER, F. H. Oats Chemistry and Technology. Saint Paul: American Association of Cereal Chemists, 1986. p. 413-426.

[23] WOOD, P. J. et al Large-scale preparation and properties of oat fractions enriched in (1-3) (1-4) - $\beta$-D-glucan. Cereal Chemistry, Saint Paul, v. 66, n. 2, p. 97-103, 1989. 